\title{
Can Ultrasound Alone Predict Papillary Thyroid Carcinoma with Desmoid-Type Fibromatosis? A Retrospective Analysis of 13 Cases, Focusing on the Stromal Area
}

\section{(ㄷ) (i) $($ ) $\odot$}

\author{
Authors

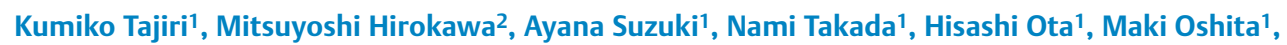 \\ Mitsuhiro Fukushima ${ }^{3}$, Kaoru Kobayashi ${ }^{3}$, Akira Miyauchi ${ }^{3}$
}

Affiliations

1 Kuma Hospital, Department of Clinical Laboratory, Kobe, Japan

2 Kuma Hospital, Department of Diagnostic Pathology and Cytology, Kobe, Japan

3 Kuma Hospital, Department of Surgery, Kobe, Japan

Key words

Papillary carcinoma, thyroid, ultrasound, Desmoid-type

fibromatosis

received $\quad 04.10 .2017$

revised 21.02.2018

accepted 05.03 .2018

\section{Bibliography}

DOI https://doi.org/10.1055/a-0591-6163

Ultrasound Int Open 2018; 4: E39-E44

(c) Georg Thieme Verlag KG Stuttgart · New York

ISSN 2199-7152

Correspondence

Ms. Kumiko Tajiri, MT

Kuma Hospital

Department of Laboratory

8-2-35, Simoyamate-dori

Chuo-ku, Kobe, 650-0011

Japan

Tel.: + 81/78/3713721

tajiri01@m.kuma-h.or.jp

\section{ABSTRACT}

Purpose Papillary thyroid carcinoma with desmoid-type fibromatosis (PTC-DTF) is extremely rare. So far, only 4 cases describing the ultrasound findings of this variant have been reported. Here, we describe the ultrasound findings of 13 cases of PTC-DTF, focusing especially on the DTF area.

Materials and Methods We retrospectively analyzed the clinical reports, ultrasound reports, and ultrasound photographs obtained from medical records at Kuma Hospital.

Results The patients included 8 women and 5 men with a mean age of 47.9 years. The widest dimension of the nodules ranged from 16 to $79 \mathrm{~mm}$ (mean: $37.5 \mathrm{~mm}$ ). The original ultrasound reports classified the nodules as either intermediate suspicion or high suspicion. A diagnosis of PTC was suspected in 12 nodules, and anaplastic carcinoma was suspected in 1 nodule. PTC-DTF presented with an irregularly shaped nodule (100\%), taller-than-wide sign (84.6\%), heterogeneous echogenicity (100\%), no microcalcification (76.9\%), and no or mild flow signal on Doppler (75.0\%). The DTF area was identified in the ultrasound photographs of 8 nodules. DTF areas were generally heterogeneous (62.5\%) and more hypoechoic (71.4\%) than PTC areas. Microcalcification was not observed in the DTF areas. All of the DTF areas revealed no or mild flow signal. On ultrasound elastography, the DTF areas were not stiff, and they were more elastic than the PTC areas.

Conclusion It is difficult to predict PTC-DTF using ultrasound alone, and B-mode ultrasonography is more reliable than ultrasound elastography in the ultrasound diagnosis of malignant thyroid nodules.

\section{Introduction}

Papillary thyroid carcinoma (PTC) has many morphologic variants. PTC with extensive proliferation of fibroblasts and myofibroblasts in the stroma is extremely rare, and has been described as "PTC with nodular fasciitis-like stroma” [1-3]. Recently, Rebecchini et al. proposed that this variant should be renamed PTC with desmoid-type fibromatosis (DTF), because the mesenchymal component showed aberrant nuclear and cytoplasmic immunoreactivity for beta-catenin and harbored a heterozygous somatic activating mutation in the corresponding CTNNB1 gene [4]. Given its rarity, reports on PTCDTF have been sporadic. To the best of our knowledge, only 4 cases describing ultrasound findings of PTC-DTF have been reported so far [4-6]. Here, we report the ultrasound findings of 13 PTC-DTF cases from a single institute, focusing especially on the DTF area. The aim of this study was to evaluate ultrasound features of PTCDTF and correlate these features with the pathological findings. 


\section{Materials and Methods}

Pathology reports of 10,659 cases of PTC resected at Kuma Hospital between January 2007 and December 2016 were reviewed. Of these, 13 cases $(0.1 \%)$ of PTC-DTF were ultimately included in this

- Table 1 Clinical and ultrasound findings of 13 cases of papillary thyroid carcinoma with desmoid-type fibromatosis.

\begin{tabular}{|c|c|c|c|}
\hline \multicolumn{2}{|l|}{ Female/male } & $8 / 5$ & \\
\hline \multicolumn{2}{|c|}{ Age [years] (mean) } & $19-77$ & $(47.9)$ \\
\hline \multicolumn{2}{|c|}{$\begin{array}{l}\text { Preoperative serum thyroglobulin } \\
{[\mathrm{ng} / \mathrm{mL}] \text { (years) }}\end{array}$} & $7.0-444.1$ & $(84.5)$ \\
\hline \multicolumn{2}{|c|}{$\begin{array}{l}\text { Antithyroglobulin antibody positive } \\
{[>39.9 \mathrm{IU} / \mathrm{mL}]}\end{array}$} & 1 & $(7.7 \%)$ \\
\hline \multicolumn{4}{|c|}{ Original ultrasound report } \\
\hline \multicolumn{2}{|c|}{ Intermediate suspicion } & 2 & $(15.4 \%)$ \\
\hline \multicolumn{2}{|c|}{ High suspicion } & 11 & $(84.6 \%)$ \\
\hline \multicolumn{2}{|c|}{ Location [left/right/isthmus] } & $5 / 7 / 1$ & \\
\hline \multicolumn{2}{|c|}{ Maximum diameter [mm] (mean) } & $16-79$ & $(37.5)$ \\
\hline \multicolumn{2}{|l|}{$\mathrm{A} / \mathrm{T}$ ratio } & $0.9-2.5$ & $(1.6)$ \\
\hline \multicolumn{2}{|c|}{$\mathrm{A} / \mathrm{T}$ ratio $>1.0$} & 11 & $(84.6 \%)$ \\
\hline \multirow[t]{3}{*}{ Shape } & Round to oval & 0 & $(0 \%)$ \\
\hline & Irregular & 13 & $(100 \%)$ \\
\hline & Spiculated & 0 & $(0 \%)$ \\
\hline \multirow[t]{4}{*}{ Margin } & Well-defined & 7 & $(53.8 \%)$ \\
\hline & Ill-defined & 6 & $(46.2 \%)$ \\
\hline & Halo & 0 & $(0 \%)$ \\
\hline & Lateral shadow & 3 & $(23.1 \%)$ \\
\hline \multirow[t]{6}{*}{ Echogenicity } & Homogeneous & 0 & $(0 \%)$ \\
\hline & Heterogeneous & 13 & $(100 \%)$ \\
\hline & Markedly hypoechoic & 5 & $(38.5 \%)$ \\
\hline & Hypoechoic & 3 & $(23.1 \%)$ \\
\hline & Isoechoic & 5 & $(38.5 \%)$ \\
\hline & Hyperechoic & 0 & $(0 \%)$ \\
\hline \multirow[t]{4}{*}{ Calcification } & $\begin{array}{l}\text { Macrocalcification } \\
(>2 \mathrm{~mm})\end{array}$ & 4 & $(30.8 \%)$ \\
\hline & $\begin{array}{l}\text { Microcalcification } \\
(<2 \mathrm{~mm})\end{array}$ & 3 & $(23.1 \%)$ \\
\hline & Peripheral calcification & 1 & $(7.7 \%)$ \\
\hline & $\begin{array}{l}\text { Marked posterior } \\
\text { shadowing }\end{array}$ & 1 & $(7.7 \%)$ \\
\hline \multirow{4}{*}{$\begin{array}{l}\text { Vascularity } \\
(n=12)\end{array}$} & No flow signal & 1 & $(8.3 \%)$ \\
\hline & Mild flow signal & 8 & $(66.7 \%)$ \\
\hline & Marked flow signal & 2 & $(16.7 \%)$ \\
\hline & Perinodular flow signal & 2 & $(16.7 \%)$ \\
\hline \multicolumn{2}{|c|}{ Acoustic posterior enhancement } & 6 & $(46.2 \%)$ \\
\hline \multicolumn{2}{|c|}{ Extrathyroidal spread } & 5 & $(38.5 \%)$ \\
\hline \multirow{2}{*}{$\begin{array}{l}\text { Suspected } \\
\text { nodal } \\
\text { metastases }\end{array}$} & Central & 5 & $(38.5 \%)$ \\
\hline & Lateral & 7 & $(53.8 \%)$ \\
\hline
\end{tabular}

Data are expressed as number (\%) or range (mean). A/T ratio, anteroposterior-to-transverse diameter ratio study. A diagnosis of PTC-DTF was made based on the presence of extensive fibroblast and myofibroblast proliferation in the stroma of PTC. PTC cases with desmoplastic changes, such as granulation tissue-like stroma, were excluded. DTF components accounted for $20 \%$ to $95 \%$ of the nodules. We then retrospectively analyzed the clinical and ultrasound reports with photographs obtained from medical records at Kuma Hospital. Ultrasound was performed using the APLIO 80 SSA-770 A (Toshiba Medical Systems Co., Ltd., Otawara, Japan) or APLIO 500 TUS-A500 (Toshiba) ultrasound machine with the PLT-805AT (Toshiba) or PLT-1005BT (Toshiba) probe. Real-time tissue elastography was performed in 2 cases using the EUB-7500 (Hitachi Medical Systems., Tokyo, Japan) ultrasound machine with the EUP-L74M (Hitachi) probe or APLIO 500 TUS-A500 ultrasound machine with the PLT-1005BT probe. Ultrasound reports were interpreted based on patterns proposed by the 2015 American Thyroid Association Management Guidelines [7]. For tissue stiffness, a 4-point scoring system proposed by Asteria et al. [8] was adopted. Statistical analysis was carried out with Fisher's exact test or Student's t-test. P-values $<0.05$ were considered statistically significant.

\section{Results}

Clinical and ultrasound findings of 13 patients with PTC-DTF are shown in $>$ Table 1 . The patients included 8 women and 5 men with a mean age of 47.9 years (range 19-77 years). Cases with a history of ionizing radiation exposure or familial polyposis were excluded. Serum thyroglobulin levels ranged from 7.0 to $444.1 \mathrm{ng} / \mathrm{mL}$ (mean: $84.5 \mathrm{ng} /$ $\mathrm{mL}$ ) and were elevated (>46.5 $\mathrm{ng} / \mathrm{ml}$ ) in 6 patients $(46.2 \%)$. Anti-thyroglobulin antibody was positive $(>39.9 \mathrm{IU} / \mathrm{mL}$ ) in 1 patient $(7.7 \%)$.

Original ultrasound reports classified the nodules as either intermediate suspicion ( 2 nodules, $15.4 \%$ ) or high suspicion (11 nodules, 84.6\%). Among them, a diagnosis of PTC was suspected in 12 nodules, and anaplastic carcinoma was suspected in 1 nodule. There were no nodules with very low or low suspicion.

5 nodules were located in the left lobe, 7 in the right lobe, and 1 in the isthmus. The widest dimension of the nodules ranged from 16 to $79 \mathrm{~mm}$ (mean: $37.5 \mathrm{~mm}$ ). The mean anteroposterior-to-transverse diameter $(\mathrm{A} / \mathrm{T})$ ratio was 1.6 (range: $0.9-2.5$ ), and the tallerthan-wide sign ( $A / T$ ratio $>1.0$ ) was detected in 11 nodules $(84.6 \%)$ ( $\triangleright$ Fig. 1). All of the nodules were irregularly shaped ( $\triangleright$ Fig. 1), and no spiculated nodules were observed. The margins were either well-defined (7 nodules, $53.8 \%$ ) or ill-defined (6 nodules, $46.2 \%$ ). A thin, sonolucent rim surrounding the thyroid nodule (halo, marginal hypoechoic zone) was not observed in any case. Three nodules (23.1\%) exhibited a lateral shadow. Echogenicity was heterogeneous in all nodules ( $>$ Fig. 2 ), and the predominant echogenicity was markedly hypoechoic in 5 nodules (38.5\%), hypoechoic in 3 nodules (23.1\%), and isoechoic in 5 nodules (38.5\%). No hyperechoic nodules were observed. Macrocalcification ( $>2 \mathrm{~mm}$ ) and punctate microcalcification $(<2 \mathrm{~mm}$ ) was observed in 4 nodules (30.8\%) and 3 nodules (23.1\%), respectively. One nodule $(7.7 \%)$ showed peripheral calcification ("eggshell" calcification), and 1 nodule $(7.7 \%)$ showed marked posterior shadowing. Twelve nodules were examined by Doppler ultrasound, $75.0 \%$ of which showed no or a mild flow signal. Acoustic posterior enhancement was observed in 6 nodules (46.2\%), and 5 nodules (38.5\%) showed ex- 


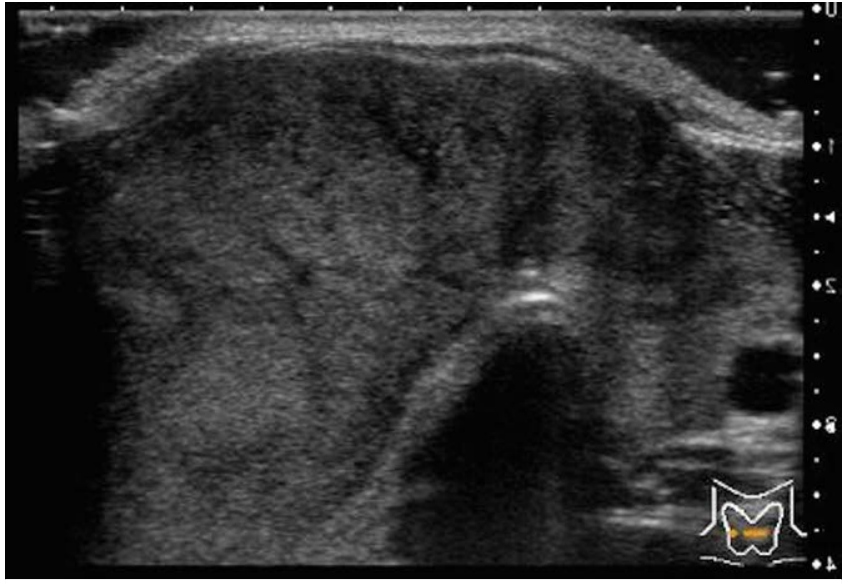

- Fig. 1 Papillary thyroid carcinoma with desmoid-type fibromatosis. Irregularly shaped, well-defined, isoechogenic nodule with a taller-than-wide sign (B-mode, transverse view).

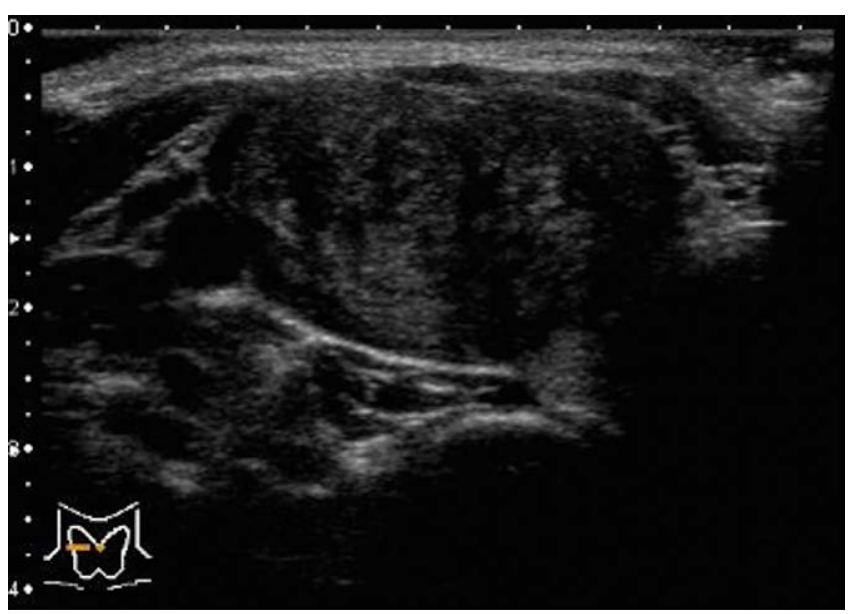

Fig. 2 Papillary thyroid carcinoma with desmoid-type fibromatosis. Echogenicity of the nodule is hypoechogenic and heterogeneous (B-mode, transverse view).

trathyroidal spread. Central and lateral nodal metastases were suspected in 5 nodules (38.5\%) and 7 nodules (53.8\%), respectively.

Based on the macro- and microscopic observations of the resected nodules, DTF and PTC areas were identified on the ultrasound images in 8 nodules and 7 nodules, respectively ( $>$ Table 2 ). DTF areas were homogeneous in 3 nodules (37.5\%) ( $>$ Fig. 3a) and heterogeneous in 5 nodules (62.5\%) ( $\mathbf{F i g}$. 4a). Compared with PTC areas, 2 DTF areas (28.6\%) were more hyperechoic ( $>$ Fig. $5 a$ ) and 5 DTF areas (71.4\%) were more hypoechoic ( $\mathbf{F i g}$. 3a). Microcalcification was not observed in any DTF area. All of the DTF areas revealed no or a mild flow signal ( $\mathbf{F i g . 6 a ) . ~ U l t r a s o u n d ~ e l a s t o g r a p h y ~}$ was performed in cases 7 and 13 . In case 13, a large part of the DTF area that occupied the majority of the tumor was somewhat elastic (class 2) ( $\mathbf{F i g . ~ 4 c ) . ~ I n ~ c a s e ~ 7 , ~ t h e ~ D T F ~ a r e a ~ w a s ~ e l a s t i c ~ ( c l a s s ~} 1$ ), and the PTC area was somewhat elastic (class 2) (॰ Fig. 7).
- Table 2 Ultrasound findings of desmoid-type fibromatosis and papillary thyroid carcinoma areas in 13 cases of papillary thyroid carcinoma with desmoid-type fibromatosis.

\begin{tabular}{|c|c|c|}
\hline Parameter & $\begin{array}{c}\text { DTF area } \\
\text { (n=8) No. of } \\
\text { nodules }(\%)\end{array}$ & $\begin{array}{c}\text { PTC area } \\
\text { (n=7) No. of } \\
\text { nodules }(\%)\end{array}$ \\
\hline Echogenicity & & \\
\hline Homogeneous & $3(37.5 \%)$ & $0(0 \%)$ \\
\hline Heterogeneous & $5(62.5 \%)$ & $7(100 \%)$ \\
\hline Markedly hypoechoic & $3(37.5 \%)$ & $0(0 \%)$ \\
\hline Hypoechoic & $3(37.5 \%)$ & $4(57.1 \%)$ \\
\hline Isoechoic & $2(25.0 \%)$ & $3(42.9 \%)$ \\
\hline Hyperechoic\# $(\mathrm{n}=7)$ & $2(28.6 \%)$ & \\
\hline Hypoechoic\# $(\mathrm{n}=7)$ & $5(71.4 \%)$ & \\
\hline Calcification & & $1(14.3 \%)$ \\
\hline Macrocalcification $(>2 \mathrm{~mm})$ & $1(12.5 \%)$ & $2(28.6 \%)$ \\
\hline Microcalcification $(<2 \mathrm{~mm})$ & $0(0 \%)$ & \\
\hline Vascularity & & $2(28.6 \%)$ \\
\hline No flow signal & $1(12.5 \%)$ & $3(42.9 \%)$ \\
\hline Mild flow signal & $7(87.5 \%)$ & $1(14.3 \%)$ \\
\hline Marked flow signal & $0(0 \%)$ & \\
\hline Elastography\#\# $(\mathrm{n}=2)$ & $1(50.0 \%)$ & \\
\hline Class 1 & $1(50.0 \%)$ & \\
\hline Class 2 & & \\
\hline
\end{tabular}

Data are expressed as number (\%). \#; compared with PTC areas $(n=7)$

\#\#train elastographic class

DTF: desmoid-type fibrosis; PTC: papillary thyroid carcinoma

\section{Discussion}

To the best of our knowledge, only 4 cases describing ultrasound findings of PTC-DTF have been reported so far [4-6]. In 2010, Lee et al. [5] first described the ultrasound findings of a PTC-DTF case in which the nodule showed homogeneous hypoechogenicity with a circumscribed margin; color Doppler ultrasound showed minimal vascular flow in the nodule. The nodule in a case reported by $\mathrm{Na}$ et al. revealed a mixed echoic pattern composed of markedly and slightly hypoechoic portions [6]; the markedly hypoechoic portion showed increased blood flow. Rebecchini et al. described ultrasound findings of 2 PTC-DTF cases [4]; one of the nodules was partially cystic with sparse microcalcification and a central hyperechoic zone, and the other had an ill-defined border with no microcalcification. Thus, characteristic ultrasound findings of DTF components have not been well established.

In our study, PTC-DTF sonographically exhibited an irregularly shaped nodule with heterogeneous echogenicity and a taller-thanwide sign. The predominant echogenicity was markedly hypoechoic, hypoechoic, or isoechoic. The incidence of punctate microcalcification was low $(23.1 \%$ ) and $75.0 \%$ of the nodules showed no or a mild flow signal. The ultrasound findings of PTC-DTF did not appear to be 

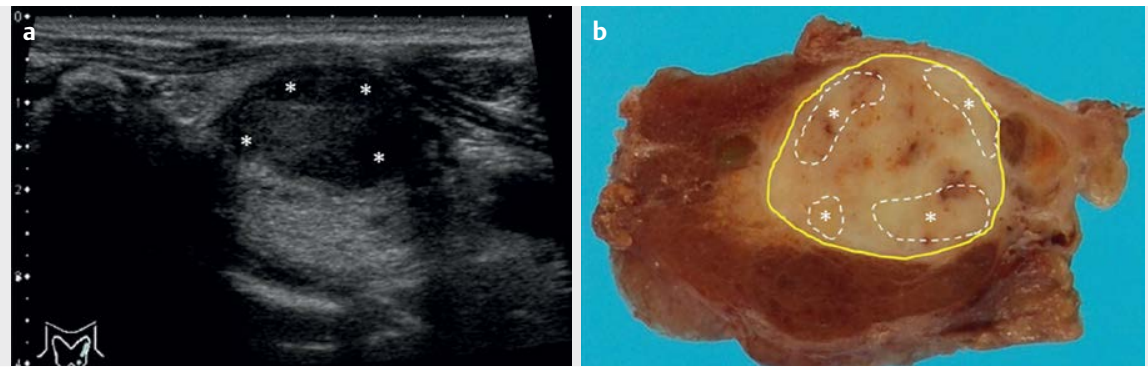

-Fig. 3 Papillary thyroid carcinoma with focal desmoid-type fibromatosis (DTF). The DTF area (*; areas surrounded by white dotted line) was homogeneous and more hypoechoic than the PTC area. (a; B-mode, longitudinal view, right; b; cut surface of resected thyroid).
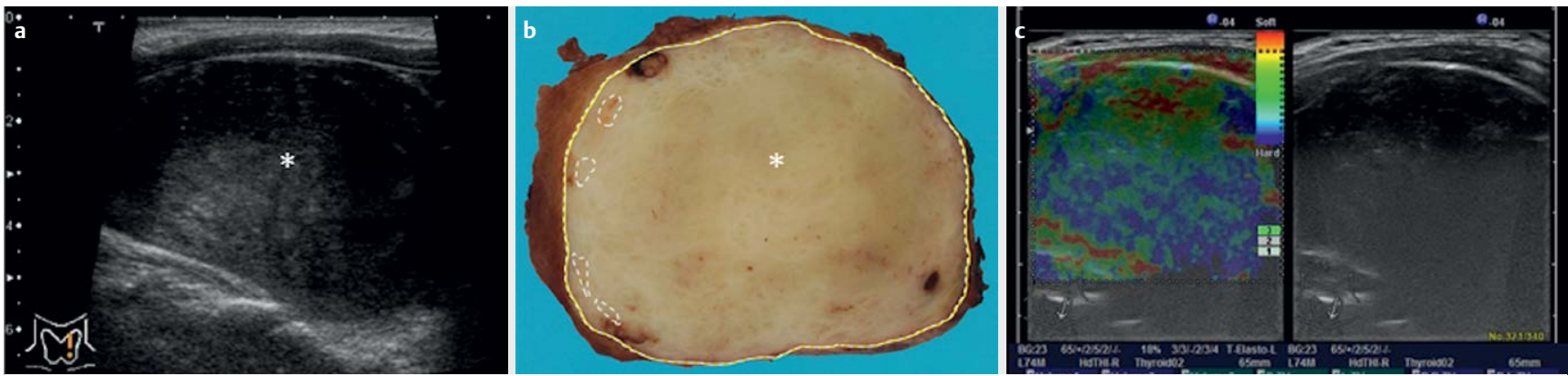

- Fig. 4 Papillary thyroid carcinoma with a massive desmoid-type fibromatosis (DTF) area. The echogenicity of DTF area ( ${ }^{*}$; area surrounded by white dotted line) was heterogeneous and interpreted as being strain elastography class 2. (a; B-mode, longitudinal view, b; cut surface of resected thyroid, c; elastography).
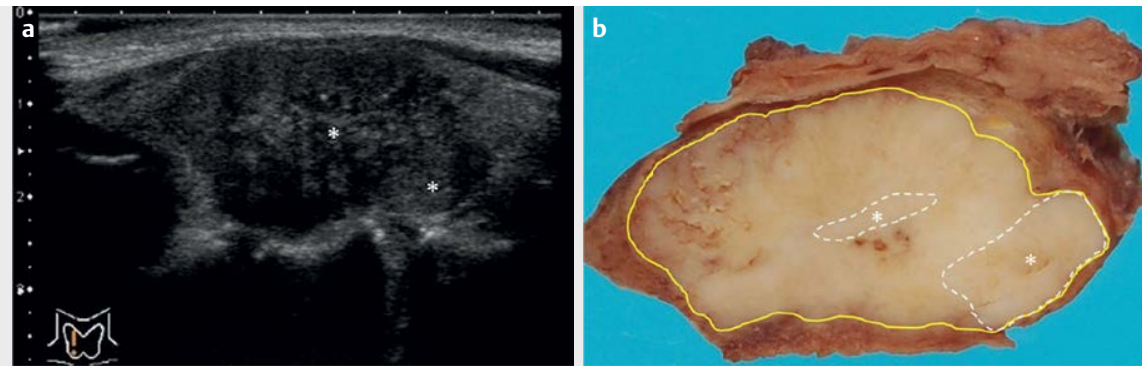

-Fig. 5 Papillary thyroid carcinoma (PTC) with a focal desmoid-type fibromatosis (DTF) area. The DTF area (*; areas surrounded by white dotted line) was more hyperechoic than the PTC area. Punctate microcalcification was seen in the PTC area. (a; B-mode, longitudinal view, b; cut surface of resected thyroid).
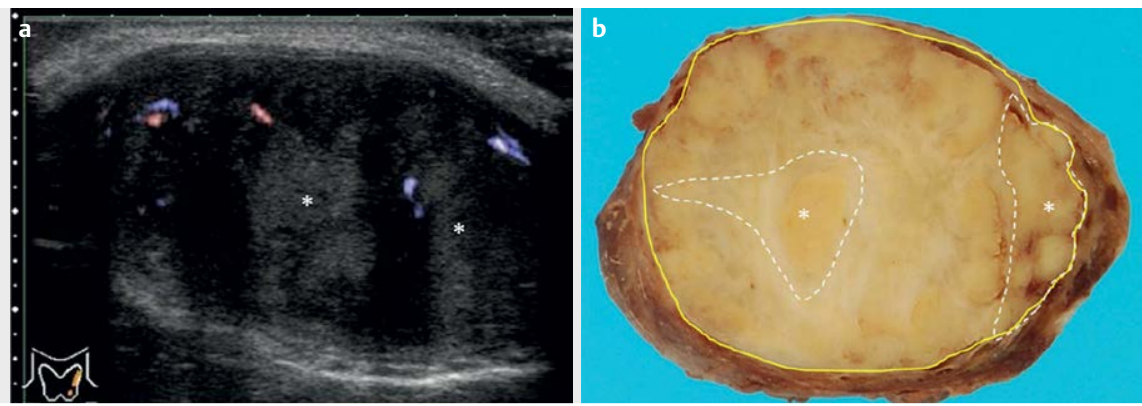

-Fig. 6 Papillary thyroid carcinoma (PTC) with focal desmoid-type fibromatosis (DTF). The DTF area ( ; ; areas surrounded by white dotted line) was homogeneous and more hyperechoic than the PTC area. No flow signal was seen. (a; B-mode, color Doppler, longitudinal view, b; cut surface of resected thyroid). 

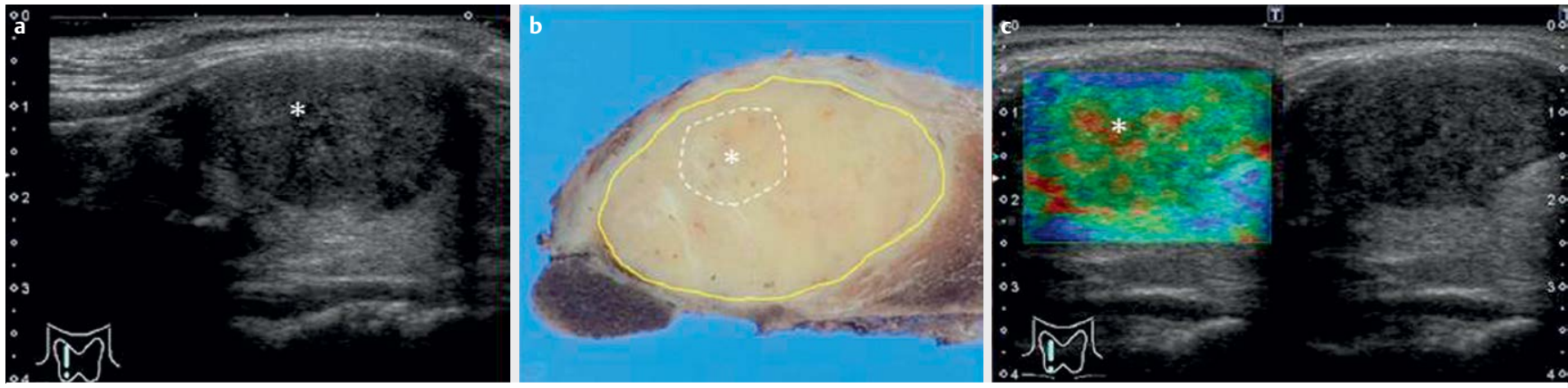

-Fig. 7 Papillary thyroid carcinoma (PTC) with focal desmoid-type fibromatosis (DTF). The DTF area (*; area surrounded by white dotted line) was more hypoechoic than the PTC area. The strain elastography classes of the DTF and PTC areas were 1 and 2, respectively. (a; B-mode, longitudinal view, $\mathbf{b}$; cut surface of resected thyroid, c; elastography).

much different from those of conventional PTC. As PTC-DTF is composed of both PTC and DTF components, we believe that the 2 components should be evaluated separately.

As the name implies, DTF components of PTC-DTF are microscopically similar to DTF arising in the soft tissue [9]. According to ultrasound reports of soft tissue DTF [10]-[11], masses display heterogeneous or homogeneous echogenicity and are hypoechoic, isoechoic, or hyperechoic. Most of the cases showed posterior acoustic enhancement, substantial flow, and high resistive index values $(>0.70)$. No calcifications or cystic areas were noted. In the present study, based on the macro- and microscopic findings, we could identify DTF areas on the ultrasound images in only 8 out of 13 nodules. DTF areas tended to be heterogeneous in echogenicity and more hypoechoic than PTC areas. Microcalcification was not observed. On Doppler ultrasound, the nodules revealed no or a mild flow signal, which is similar to soft tissue DTF.

Ultrasound elastography can be easily performed in the thyroid due to its superficial location $[12,13]$. However, the assessment of deeply located nodules is difficult due to the depth-related decay of the stress [14]. Azizi et al. reported that thyroid nodule stiffness measured by elastography is an independent predictor of thyroid carcinoma with a positive predictive value that is greater than or equal to that of conventional ultrasonographic characteristics [15]. We hypothesized that the DTF area would be firm based on its composition of dense collagenous connective tissue. Contrary to our expectations, the DTF areas were not stiff, and in one case (case 7), the DTC area was more elastic than the PTC area. Thus, clinicians should be aware that PTC- DTF shows a benign pattern on ultrasound elastography. In conclusion, B-mode ultrasonography is more reliable than ultrasound elastography in the ultrasound diagnosis of malignant thyroid nodules.

\section{Conclusion}

This is the first description of ultrasound findings of PTC-DTF, focusing especially on the DTF area. The ultrasound findings of PTCDTF include an irregularly shaped nodule, taller-than-wide sign, heterogeneous echogenicity, no microcalcification, and no or a mild flow signal. DTF areas tend to be heterogeneous in echogenicity and more hypoechoic than PTC areas. Despite the presence of abundant dense connective tissue, DTF areas show a benign pat- tern on ultrasound elastography. We therefore conclude that it is difficult to predict the DTF variant of PTC using ultrasound alone. Moreover, B-mode ultrasonography is more reliable than ultrasound elastography in the ultrasound diagnosis of malignant thyroid nodules. Further analysis with a greater number of cases is needed to clarify the ultrasound findings of DTF areas of PTC-DTF nodules.

\section{Conflict of Interest}

The authors declare no conflicts of interest.

References

[1] Chan JK, Carcangiu ML, Rosai J. Papillary carcinoma of thyroid with exuberant nodular fasciitis-like stroma. Report of three cases. Am J Clin Pathol 1991; 95: 309-314

[2] Yang Y], LiVolsi VA, Khurana KK. Papillary thyroid carcinoma with nodular fasciitis-like stroma. Pitfalls in fine-needle aspiration cytology. Arch Pathol Lab Med 1999; 123: 838-841

[3] Ginter PS, Scognamiglio T. Papillary thyroid carcinoma with nodular fasciitis-like stroma: A usual entity with distinctive morphology. Int J Surg Pathol 2015; 23: 305-307

[4] Rebecchini C, Nobile A, Piana S, Sarro R, Bisig B, Gerasioms SP, Saglietti C, Matter M, Marino L, Bongiovanni M. Papillary thyroid carcinoma with nodular fasciitis-like stroma and $\beta$-catenin mutations should be renamed papillary thyroid carcinoma with desmoid-type fibromatosis. Mod Pathol 2017; 30: 236-245

[5] Lee KW, Park WC, Kim JY, Lee YS, Kim MK. Sonographic finding of papillary thyroid carcinoma with nodular fasciitis-like stroma. J Med Ultrason 2010; 37: 59-61

[6] Na KY, Kim HS, Sung JY, Park WS, Kim YW. Papillary carcinoma of the thyroid gland with nodular-fasciitis-like stroma. Korean J Pathol 2013; 47: $167-171$

[7] Haugen BR, Alexander EK, Bible KC, Doherty GM, Mandel SJ, Nikiforov YE, Pacini F, Randolph GW, Sawka AM, Schlumberger M, Schuff KG, Sherman SI, Sosa JA, Steward DL, Tuttle RM, Wartofsky L. 2015 American thyroid association management guidelines for adult patients with thyroid nodules and differentiated thyroid cancer: The American thyroid association guidelines task force on thyroid nodules and differentiated thyroid cancer. Thyroid 2016; 26: 1-133 
[8] Asteria C, Giovanardi A, Pizzocaro A, Cozzaglio L, Morabito A, Somalvico F, Zoppo A. US-elastography in the differential diagnosis of benign and malignant thyroid nodules. Thyroid 2008; 18: 523-531

[9] Takada N, Hirokawa M, Ito M, Ito A, Suzuki A, Higuchi M, Kuma S, Hayashi T, Kishikawa M, Horikawa S, Miyauchi A. Papillary thyroid carcinoma with desmoid-type fibromatosis: A clinical, pathological, and immunohistochemical study of 14 cases. Endocr J In press, (Advance Publication)

[10] Wang Y, Cui NY, Li L, Zhang R, Hao YZ, Xue Li-Yan, Zhou CZ, jiang YX. An abdominal desmoid-type fibromatosis. Quant Imaging Med Surg 2013; 3: 228-230

[11] Lou L, Teng J, Qi H, Ban Y. Sonographic appearances of desmoid tumors. J Ultrasound Med 2014; 33: 1519-1525

[12] Dietrich CF, Barr RG, Farrokh A, Dighe M, Hocke M, Jenssen C, Dong Y, Saftoiu A, Havre RF. Strain elastography - how to do it? Ultrasound Int Open 2017; 3: E137-E149
[13] Cantisani V, Grazhdani H, Drakonaki E, D'Andrea V, Di Segni M, Kaleshi E, Calliada F, Catalano C, Redler A, Brunese L, Drudi FM, Fumarola A, Carbotta G, Frattaroli F, Di Leo N, Ciccariello M, Caratozzolo M, D'Ambrosio F. Strain US elastography for the characterization of thyroid nodules: Advantages and limitation. Int J Endocrinol. 2015; 2015: 908575

[14] Tranquart F, Bleuzen A, Pierre-Renoult P, Chabrolle C, Sam Giao M, Lecomte P. Elastosonography of thyroid lesions. J Radiol 2008; 89: 35-39

[15] Azizi G, Keller J, Lewis M, Puett D, Rivenbark K, Malchoff C. Performance of elastography for the evaluation of thyroid nodules: A prospective study. Thyroid 2013; 23: 734-740 